\title{
émulations
}

\section{Agnès Roche - Des vies de pauvres. Les classes populaires dans le monde rural}

\author{
Alicia Rinaldy \\ Émulations - Revue de sciences sociales \\ 2018, «Comptes rendus critiques, En ligne»
}

\section{Article disponible à l'adresse suivante}

https://ojs.uclouvain.be/index.php/emulations/article/view/7423

\section{Pour citer cet article}

Alicia Rinaldy, «Agnès Roche — Des vies de pauvres. Les classes populaires dans le monde rural », Émulations, en ligne. Mise en ligne le 25 mai 2018.

DOI : 10.14428/emulations.cr.042

Distribution électronique : Université catholique de Louvain (Belgique) : ojs.uclouvain.be

(C) Cet article est mis à disposition selon les termes de la Licence Creative Commons Attribution, Pas d'Utilisation Commerciale 4.0 International. http://creativecommons.org/licenses/by-nc/4.0/

Éditeur : Émulations - Revue de sciences sociales / Presses universitaires de Louvain https://ojs.uclouvain.be/index.php/emulations

ISSN électronique : 1784-5734

UCL PRESSES

UNIVERSITAIRES 


\title{
Agnès Roche - Des vies de pauvres. Les classes populaires dans le monde rural
}

\begin{abstract}
Alicia Rinaldy ${ }^{1}$
Recensé : Agnès Roche, Des vies de pauvres. Les classes populaires dans le monde rural, Rennes, Presses Universitaires de Rennes, 2016, 340 p.

La publication en novembre 2016 de l'ouvrage d'Agnès Roche, Des vies de pauvres. Les classes populaires dans le monde rural s'inscrit en France dans une actualité brûlante. Les affects de la politique immédiate s'invitent immanquablement dans la réception que nous pouvons avoir aujourd'hui de ce texte.

Si l'on en croit les commentateurs et les articles de presse qui ont été publiés au lendemain du premier tour de l'élection présidentielle de 2017, les voix des classes populaires rurales seraient majoritairement allées au Front National parce qu'elles se considèrent comme les victimes d'un déclassement social, reléguées dans des territoires périphériques en proie au chômage de masse ${ }^{2}$. Ce constat n'est pas nouveau ni tout à fait faux, mais la réalité semble pourtant plus complexe ${ }^{3}$. Bien que le champ politique soit le point aveugle de l'étude, qui s'intéresse davantage aux conditions économiques, familiales et scolaires de (re)production de ces classes laborieuses, elle donne cependant à entendre longuement ces voix, que l'on a finalement peu entendues lors de cette récente séquence médiatique et politique.
\end{abstract}

Alors qu'Emmanuel Macron, nouvellement élu, a annoncé à la mi-juillet 2017 la baisse de cinq euros de l'Aide Personnalisée au Logement (APL), dont bénéficie une

${ }^{1}$ Jeune docteure associée au Centre de Recherche et de Documentation sur les Amériques (CREDA-UMR 7227) et chargée de cours à la Universidad Nacional Autónoma de México (UNAM) et à l'Universidad Autónoma de la Ciudad de México (UACM).

${ }^{2}$ Voici quelques articles parus dans la presse à l'époque : Delphine Bancaud, « Présidentielle : Un vote rural pour Marine Le Pen et un vote plus urbain pour Emmanuel Macron », $20 \mathrm{mi}-$ nutes, publié le 24/04/17, URL : http://www.20minutes.fr/elections/presidentielle/2055759 20170424-presidentielle-vote-rural-marine-pen-vote-plus-urbain-emmanuel-macron ;

«Exode urbain et inégalités : les cartes du vote FN », France Culture, publié le 24/04/2017, URL : https://www.franceculture.fr/politique/exode-urbain-et-inegalites-les-cartes-du-votefn; Baptiste Langlois, "Ruralité. Plus il y a de services, moins le vote FN est fort », Ouest France, publié le 16/03/2017, URL : http://www.ouest-france.fr/societe/ruralite-plus-il-y-deservices-moins-le-vote-fn-est-fort-4860928; Gautier Lecardonnel, «FN : un vote rural? », Courrier picard, publié le 01/05/2017, URL: http://www.courrier-picard.fr/27470/article/2017-05-01/fn-un-vote-rural.

${ }^{3}$ Dans une très récente publication, Violaine Girard propose de reconsidérer et de nuancer ce cliché (Girard, 2017). 
grande partie des enquêtés, l'ouvrage montre à quel point cette politique publique n'est pas destinée exclusivement aux étudiants; il documente précisément ce que représente ce manque à gagner dans un budget familial souvent très serré. On découvre au fil des pages que les quelques subsides versés par l'État (comme le RSA, le minimum vieillesse, l'aide au retour à l'emploi, l'allocation de solidarité spécifique ou encore l'allocation aux adultes handicapés) s'avèrent absolument nécessaires pour simplement prétendre pouvoir survivre. Ainsi, ce couple, Corinne et Gérard, qui tant bien que mal parvient chaque mois à nourrir huit âmes avec 2400 euros ; Jean-Yves, qui perçoit une retraite de 780 euros et qui avoue devoir emprunter parfois quelques dizaines d'euros à son fils; Solange qui bricole avec 100 euros une fois le loyer et les charges payés; ou encore Cindy, cette jeune mère de 23 ans, qui vit avec 680 euros et qui fait « hyper gaffe quand [elle fait ses] courses » (Roche, 2016 : 56). Comme Fanny le résume, il n’y a en général dans ces familles « pas de plaisir, à part le tabac » (Roche, 2016: 309).

L'enquête d'Agnès Roche a été menée en Auvergne, dans le département du Puy-deDôme, où près de la moitié des foyers fiscaux est non imposable, où le revenu médian s'élève à 17000 euros, où $17 \%$ de ses habitants vivent en dessous du seuil de pauvreté (977 euros par mois pour une personne seule) et où le taux de chômage était de 10,2 \% en 2012 (similaire à la moyenne nationale). Au sein du département, l'aire urbaine de Clermont-Ferrand tend à tirer vers le haut ces moyennes qui sont plus préoccupantes encore lorsqu'elles recouvrent les circonscriptions rurales, à l'endroit même où l'étude a été effectuée. Ainsi, dans les Combrailles, au nord-ouest de la capitale du département, $63 \%$ des foyers fiscaux sont non imposables, et le revenu médian est de 14600 euros; dans le Livradois-Forez (à l'est), 23,5\% des ménages se situent sous le seuil de pauvreté (2009); dans la région de moyenne montagne du Sancy, le canton de La Tour d'Auvergne est particulièrement pauvre avec un revenu médian qui s'élève à 13734 euros, $22,5 \%$ de logements indignes et 34,45 \% des ménages sous le seuil de pauvreté; enfin, les cantons de la plaine de La Limagne ont des statistiques plus favorables parce qu'ils sont moins isolés et en voie de périurbanisation (Roche, 2016 : 25-32).

Le livre d'Agnès Roche met des visages et des histoires sur ces chiffres. Il actualise le récit de la pauvreté dans la région, dont le roman d'Antoine Sylvère, Toinou. Le cri d'un enfant auvergnat, publié dans les années 1980, avait auparavant l'exclusivité. S'inscrivant dans la continuité du mouvement littéraire réaliste, l'ouvrage se centrait essentiellement sur le monde paysan. Du côté des sciences sociales, la pauvreté dans les espaces ruraux reste finalement un sujet peu étudié. Il n'existait auparavant aucune recherche sur ce thème dans la région. Au niveau national, l'auteure fait référence à l'ouvrage collectif de l'association des ruralistes français (Maclouf, 1986), publié il y a déjà plus de trente ans, ou au texte plus récent d'Alexandre Pagès (2004), qui aborde la pauvreté, mais exclusivement du point de vue des travailleurs sociaux. En somme, « on peut dire sans forcer le trait qu'il n'existe aucune publication de recherche récente sur [ce] thème d'étude» (Roche, 2016 : 20). 
Le dispositif narratif de l'ouvrage peut surprendre. Il se structure autour de deux grandes parties inégales dans leur longueur. Une première section se compose de multiples portraits subdivisés en quatre catégories de bénéficiaires de l'aide sociale. Le matériel ici présenté est imposant : il s'agit au total de 96 récits (regroupant souvent plusieurs acteurs), et on imagine des centaines d'heures d'entretiens retranscrits. À cet égard, on peut d'ailleurs envisager que ces 96 portraits puissent constituer une base de données qualitative dans laquelle pourront puiser de futures recherches. Au cours de ces 193 pages - quasiment les deux tiers du livre -, on découvre les histoires de jeunes, de retraités, de travailleurs pauvres et de paysans. L’analyse de ces catégories d'acteurs contribue à rompre avec l'idée de la campagne comme le lieu où l'agriculture est prédominante. Les paysans ne sont plus, depuis longtemps déjà, la seule image possible des ruralités (Mendras, 1967).

L'ouvrage ne prétend bien sûr pas fournir un récit exhaustif de ces trajectoires, et c'est une gageure de la part de l'auteure d'être parvenue à nous en livrer une image fine, bien que partielle. Le portrait tâche à chaque fois de revenir sur les liens familiaux, les ressources économiques, les itinéraires scolaires, les mobilités résidentielles ou les problèmes médicaux des individus. Mais on regrette parfois que certains éléments ne soient pas plus développés : plus de quatre-vingts années d'existence sont ainsi résumées en une ou deux pages; certains portraits se centrent davantage sur les parcours scolaires, alors que d'autres se structurent en priorité autour des liens familiaux, sans nous préciser la raison de ce choix. Si la méthodologie des entretiens biographiques est clairement exposée en introduction, il eut été intéressant d'expliciter pourquoi les narrations suivent tel chemin, insistent sur tel fait ou négligent tel aspect.

Malgré cet écueil inhérent à l'exercice, cette première partie purement descriptive renferme une puissante dimension heuristique pour le lecteur. Elle permet d'abord de le mettre en situation d'enquête, dans une posture compréhensive, d'écoute et de respect. C'est le propre de la recherche sociologique; il est rare néanmoins de trouver des ouvrages qui parviennent à faire coïncider l'expérience de lecture avec celle du travail de terrain. Ensuite, l'accumulation de cas permet peu à peu de dégager les invariants de ces trajectoires souvent difficiles. Chaque portrait n'a en aucun cas valeur de représentativité, mais leur répétition et comparaison mettent en lumière ce qui semble toucher indistinctement l'ensemble des parcours individuels, le commun des destinées sociales, tout en soulignant les singularités de chacun des itinéraires recueillis. Ainsi, on retrouve pêle-mêle des logements insalubres, une insécurité de l'emploi, des violences conjugales, l'entraide familiale ou au contraire la rupture des liens, des soins de santé inlassablement repoussés, des difficultés scolaires ou des addictions. Dans ce dispositif, c'est le lecteur qui tisse lui-même ces liens entre les trajectoires et qui en propose une interprétation. Ensuite, il pourra confronter sa perception de ces bribes de vie à l'analyse qu'en fait l'auteure dans la deuxième partie de l'ouvrage. Enfin, chacune des his- 
toires qui nous est donnée à lire contribue à « lutter contre l'idée, jamais vraiment assumée, mais bien souvent suggérée, que seuls les bourgeois ont une vie intéressante, digne d'intérêt » (Roche, 2016 : 24).

La deuxième partie se consacre à l'analyse que propose l'auteure de ces «vies de pauvres » et nous donne à nouveau accès à de multiples citations d'entretiens. Elle reprend d'abord les quatre catégories de publics de l'aide sociale pour y décrire les spécificités de la pauvreté pour chacune d'entre elles. Elle s'intéresse ensuite aux institutions qui les reproduisent : l'école, le travail, la famille ou encore l'aide sociale elle-même. On y apprend que le travail n'est finalement plus la grande matrice des biographies, et que l'on peut très bien travailler beaucoup sans nécessairement parvenir à sortir de sa condition de pauvre; que les liens familiaux sont souvent plus subis qu'il ne s'agit d'une véritable "protection rapprochée », comme la nommait Robert Castel (Castel, 2003); que l'innocence et l'irresponsabilité de la jeunesse sont des privilèges des classes supérieures; ou que les multiples « secousses » de l'existence (maladies, divorces, échec scolaire, chômage, etc.) frappent plus fortement encore les individus déjà fragilisés économiquement.

Dès le titre, le mot «pauvre » attire l'attention du lecteur, mais on achève la lecture sans vraiment comprendre ce que ce vocable referme. Le choix des enquêtés s'est fait sur la définition des organismes étatiques de groupes de population caractérisés par ses revenus ou ses carences. Est ainsi défini comme " pauvre » celui que la société désigne comme tel et qui bénéficie à ce titre de l'assistance. La pauvreté est alors entendue comme une construction sociale formée à partir des relations entre les individus et l'État social. Mais la pauvreté est aussi une catégorie subjective. Le protocole de recherche invitait d'ailleurs à demander à la fin des entretiens si les enquêtés se sentaient "pauvres ». Différentes réponses ont été récoltées. Certains s'approprient le mot, d'autres le relativisent, d'autres encore le refusent, comme Thomas : « Ben si on parle de sous, je pense que je n'ai pas un niveau de vie très très haut... après si on parle d'humanité, je me sens pas pauvre du tout, je me sens même plutôt riche » (Roche, 2016: 318). Cette multiplicité de réponses révèle peut-être que « la distinction », c'est-à-dire la lutte pour la différenciation sociale, n'est pas l'apanage des classes bourgeoises, pour parler en termes bourdieusiens (Bourdieu, 1979), et qu'on ne peut se limiter à une définition économique de la pauvreté.

Au moment de refermer le livre, on se demande alors si définir une personne par son manque, ce n'est pas lui faire violence une deuxième fois, et s'il n'aurait pas fallu rompre plus clairement avec la catégorisation établie par les aides publiques, structurer le texte autour des institutions qui contribuent à reproduire ces différentes formes de désaffiliation, ou encore insister davantage sur les pratiques que les classes populaires mettent en œuvre pour « s'en sortir». Dans cette perspective, les études sur le " capital d'autochtonie » peuvent par exemple constituer une piste féconde. Les travaux de Nicolas Renahy (2010), dans la continuité de ceux ouverts par Jean-Noël Retière 
(2003), ont participé à mettre en lumière ce concept ${ }^{4}$, construit à partir de l'analyse des classes populaires et ouvrières en milieu rural. Dernièrement, en mai 2016, les Presses universitaires de Rennes ont également publié un ouvrage coécrit par de jeunes chercheurs sur des travaux récents ou toujours en cours autour de cette notion (Aunis, Benet, Mege, Prat, 2016). Le " capital d'autochtonie " permet de rendre compte des ressources tirées par l'appartenance à un lieu. Il s'agit ainsi de penser « une notoriété acquise et entretenue sur un territoire singulier [...] permettant de se positionner avantageusement sur différents marchés (politique, du travail, matrimonial, associatif, etc.) » (Renahy, 2010 : 9). La notion est particulièrement opérante pour des terrains d'enquête en milieu rural où l'interconnaissance, le contrôle social, l'intersection des espaces professionnels et familiaux sont prégnants. En ce sens, l'appartenance locale semble être une entrée prometteuse pour ne pas considérer les classes populaires exclusivement sous le prisme de ce qu'elles n’ont pas, dans la négativité.

Malgré cette réserve, une telle publication était nécessaire, car il se révélait urgent de documenter avec précision et par le bas, à travers un important corpus de témoignages, les vécus des classes populaires rurales.

\section{Bibliographie}

Aunis É., Benet J., Mege A., Prat I. (dir.) (2016), Les territoires de l'autochtonie. Penser la transformation des rapports sociaux au prisme du «local», Rennes, Presses universitaires de Rennes.

Bourdieu P. (1982 [1979]), La Distinction. Critique sociale du jugement, Paris, Les Éditions de Minuit («Le Sens commun »).

CASTEL R. (2003), L’insécurité sociale. Qu'est-ce qu'être protégé ?, Paris, Seuil.

CHAmboredon J.-C. (1982), « La diffusion de la chasse et la transformation des usages sociaux de l'espace rural », Études rurales, n 87-88, p. 233-260.

GIRARD V. (2017), Le vote FN au village. Trajectoires de ménages populaires du périurbain, Paris, Éditions du Croquant (« Sociopo »).

MAClouf P. (dir.) (1986), La pauvreté dans le monde rural, Paris, l’Harmattan

\footnotetext{
${ }^{4}$ Nicolas Renahy cite aussi les travaux de l'équipe de recherche de Jean-Claude Chamboredon comme particulièrement importants dans la genèse de la notion. À partir de l'étude d'une pratique sociale telle que celle de la chasse, il va repenser la relation ville-campagne et mettre en évidence comment la chasse devient un trait constitutif de l'appartenance des "locaux» au lieu, face aux urbains qui la pratiquent comme un loisir récréatif (Chamboredon, 1982).
} 
MENDRAS H. (1967), La fin des paysans : innovations et changements dans l'agriculture française, Paris, SEDEIS.

PAgES A. (2004), La pauvreté en milieu rural, Toulouse, Presses Universitaires du Mirail.

RENAHY N. (2010), « Classes populaires et capital d'autochtonie. Genèse et usages d'une notion », Regards Sociologiques, $n^{\circ}$ 40, p. 9-26.

RETIERE J.-N. (2003), « Autour de l'autochtonie. Réflexions sur la notion de capital social populaire », Politix, nº 63, p. 121-143.

SYlvere A. (1980), Toinou. Le cri d’un enfant auvergnat, Paris, Plon (« Terre Humaine »). 